\title{
BMJ Open Changes in the upper airway following induction of anaesthesia: a prospective observational study protocol to determine the use of ultrasound in the assessment of a difficult airway in China
}

\author{
Fang Dong, ${ }^{\oplus 1}$ Yong Wang, ${ }^{2}$ Xia Wang, ${ }^{1}$ Huanyi Zhao, ${ }^{1}$ Wuhua $\mathrm{Ma}^{2}$
}

To cite: Dong F, Wang $Y$, Wang $X$, et al. Changes in the upper airway following induction of anaesthesia: a prospective observational study protocol to determine the use of ultrasound in the assessment of a difficult airway in China. BMJ Open 2019;9:e029782. doi:10.1136/ bmjopen-2019-029782

- Prepublication history for this paper is available online. To view these files, please visit the journal online (http://dx.doi org/10.1136/bmjopen-2019029782).

Received 14 February 2019 Revised 14 May 2019 Accepted 3 June 2019

\section{Check for updates}

\section{(c) Author(s) (or their} employer(s)) 2019. Re-use permitted under CC BY-NC. No commercial re-use. See rights and permissions. Published by BMJ.

${ }^{1}$ The First Clinical Medical College, Guangzhou University of Chinese Medicine, Guangzhou, China

${ }^{2}$ Department of Anaesthesiology, The First Affiliated Hospital of Guangzhou University of Chinese Medicine, Guangzhou, China

Correspondence to

Professor Wuhua Ma; gzmwh@aliyun.com

\section{ABSTRACT}

Introduction Difficult airway management is closely related to the safety and quality of medical care. However, the low incidence of correct prediction of difficult airway in clinical screening tests presents physicians with a dilemma. Depressed airway neuromuscular tension during sleep and anaesthesia tends to cause collapse of fragile parts of the upper airway. Although previous studies have confirmed that anterior cervical tissue thickness is associated with difficult airways, there is no evidence to support a correlation between a difficult airway and specific patterns or findings of anaesthesia-induced airway collapse. Thus, this study aims to examine changes in airway dimensions before and after induction of general anaesthesia to explore whether it could provide useful information regarding the specific anatomic changes occurring which may cause difficult airways.

Methods and analysis This will be a single-centre prospective observational single-blinded study, conducted in a tertiary teaching hospital in Guangzhou, China. Subjects will be recruited from patients (aged 18-65 years) scheduled for elective surgery under general anaesthesia. Sonographic measurement will be performed to detect changes in the thickness of the anterior cervical soft tissue before and after anaesthetic induction. Based on the resulting data distribution, analyses will initially compare these changes using a paired t-test or the Wilcoxon signed-rank test. The correlation of sonographic changes and Cormack-Lehane grade will be evaluated by using receiver-operating characteristic curves to detect the sensitivity and specificity of a measurement for detecting difficulties. Linear stepwise regression analysis will be used to assess the correlation between airway changes and demographic variables as well as clinical tests.

Ethics and dissemination Ethical approval has been obtained from the Ethics Committee of the First Affiliated Hospital of Guangzhou University of Chinese Medicine (ZYYECK (2018) 041). The results will be disseminated through conference presentations, professional journals and peer-reviewed publications.

Trial registration number ChiCTR1900021123; Preresults.
Strengths and limitations of this study

- No previous study has used ultrasound to investigate upper airway changes following anaesthetic induction.

- The study will explore new indicators that can provide insight into the theoretical basis for dynamic risk assessment of difficult airways.

- The trial will recruit both easy and difficult laryngoscopy subjects, which can provide information useful for evaluating the difference between difficult and normal airways.

- Patients with indications for awake and/or spontaneous breathing airway management technique will be excluded, and patients with unexpected and complicated difficult airway management will be withdrawn from the study; although this might create a potential selection bias, this will ensure patient safety and compliance with the American Society of Anesthesiologists' principle of difficult airway management.

- The repeated measurement design uses each individual as its own control, which overcomes the bias caused by individual variation; however, the influence of measurement time on observation results is not considered.

\section{INTRODUCTION}

Airway management is critical in medical intervention and is a prerequisite for successful treatment and rescue. However, in some cases, repeated attempts to perform laryngoscopy or intubation lead to lack of adequate oxygen delivery to the brain and other vital organs, which can threaten patient safety. ${ }^{1}$ In 2016, we conducted a large-scale survey of airway management in China, which showed that $73 \%$ of anaesthesiologists were concerned about difficult airways, 36\% experienced emergency airway rescue, and in $14 \%$ of hospitals, death or brain injury were caused by difficult airways. Although 
airway management-related severe complications in anaesthesia are rare, they can result in serious patient harm. ${ }^{23}$ According to the survey data obtained in the fourth National Audit Project of the Royal College of Anaesthetists and Difficult Airway Society project (NAP4), the incidence of 'cannot intubate, cannot oxygenate' is less than 1 in 5000 in general anaesthesia; however, the combined rate of death and brain damage in such cases constitutes up to $25 \%$ of all major complications. Among these adverse events, hypoxia is the most common cause of airway-related deaths. ${ }^{34}$

Obstruction of the upper airway is a relatively common complication during sleep and anaesthesia. ${ }^{5}$ Even topical anaesthesia of the oropharynx tends to increase the frequency of obstructive sleep apnoea (OSA) ${ }^{6}$ The mutual narcotic mechanism of sleep and anaesthesia is thought to trigger the bistable thalamocortical switch that controls consciousness. ${ }^{7}$ At or approaching the moment of loss of consciousness, local electromyographic activity abruptly decreases to a nadir, accompanied by an increase in mask pressure. ${ }^{8}$ Such problems often occur because the tendency for a loss of pharyngeal patency is related to anatomical factors, ${ }^{8}$ which also make intubation difficult. ${ }^{5}$

The framework of bone and cartilage on the upper airway ensures respiratory tract patency. However, the pharynx, a soft tube composed mainly of muscles and lacking in bony or cartilaginous scaffolds, provides the anatomical basis for collapse during inhalation. Due to the role of the thoracic and abdominal muscles during inhalation, the pleural cavity is enlarged, the pressure of the pleural and pharyngeal cavity is reduced, and the whole respiratory tract is in a negative pressure state. When the negative pressure exceeds the critical pressure that can be withstood by the pharyngeal airway wall, the airway may collapse, especially in individuals in whom the tract is already narrow due to compression of tissue around the pharynx. ${ }^{910}$

Accurate prediction of a difficult airway is the first step towards avoiding potential complications. Preoperative unspecified airway assessment is recommended by the American Society of Anesthesiologists (ASA) and NAP4, as well as by many international airway management guidelines. ${ }^{11}$ Whenever feasible, airway history and physical examination should be conducted in all patients; based on the anticipated likelihood of airway difficulty, such as that in patients with OSA, the ASA strongly suggests conducting additional evaluations. ${ }^{12}$

The observed incidence of unexpected difficult airways depends on the tests performed. Unfortunately, low sensitivity and high variability significantly decrease the value of the commonly used clinical screening test as a reliable clinical diagnostic approach. ${ }^{13}$ A Danish retrospective study revealed that $93 \%$ of difficult intubation and $94 \%$ of difficult mask ventilation cases were unanticipated; at the same time, only $25 \%$ and $22 \%$, respectively, of the anticipated cases actually occurred. ${ }^{14}$ A meta-analysis $^{15}$ and systematic review ${ }^{16}$ have found that both single predictors and combined tests are characterised by limited discriminative capability, sensitivity and positive likelihood ratio.

The invisible anatomic lesions (eg, vocal fold cysts) that may lead to difficult airways could be challenging to identify in standard preoperative examinations; additionally, quantitative analysis of the obstacles cannot be implemented based on clinical signs and symptoms. ${ }^{17}$ Various assistive techniques such as endoscopy, ${ }^{18} \mathrm{X}$-ray imaging, ${ }^{19} \mathrm{CT}^{20}{ }^{21}$ and $\mathrm{MRI}^{22}{ }^{23}$ have been used to image both intrinsic and extrinsic pathological changes in the trachea. ${ }^{24}$ In addition, combining the advantages of endoscopy and CT, the novel application of virtual endoscopy in anaesthesia provides a three-dimensional (3D) 'fly-through' video of intratracheal anatomy through reconstructed CT images. ${ }^{25}$ Compared with other supplementary measurements, ultrasound is an effective, non-invasive, widely available evaluation modality, which requires no radiation and which can be performed with little or no patient preparation. Furthermore, it would be a relatively objective technique and familiar to anaesthesiologists who often conduct ultrasound-guided nerve block and vascular access, as well as airway management-related operations (eg, confirmation of endotracheal tube placement and localisation of cricothyroid membrane). ${ }^{26}$ This measurement allows evaluation of adjacent soft tissue structures around the collapsible segment, such that narrowing and flaccidity can be delineated. A strong correlation has been found between the CormackLehane score and ultrasound-determined depth from the skin to the trachea at the level of the thyrohyoid membrane. ${ }^{27}$ The use of ultrasound is similar to that of the upper lip bite test, which is the optimal bedside test for predicting difficult intubation. ${ }^{13} 2628$

However, not all difficult tracheal intubations are related to obvious lesions; they can also be derived from more subtle anatomical tendencies controlled by muscular tone. ${ }^{529}$ Depression of the electromyographic activity of pharyngeal dilator muscles, as well as reduction of longitudinal tension due to reduced lung volume during anaesthesia and sleep, cannot offset the intraluminal negative pressure during inspiration and the extraluminal negative pressure from soft tissue and bone, thus leading to the occurrence of airway collapse. ${ }^{30}$ The vulnerable compliant segments are predisposed to partial or complete obstruction only when neuromuscular activation is depressed by a pharmaceutical agent ${ }^{8}$ or when sleep occurs. ${ }^{29}$ For this reason, obstruction during sleep and anaesthesia can be identified in only few patients when they are awake. ${ }^{9}$ Therefore, preoperative ultrasound examination may not be able to reveal the impact of dynamic changes on airflow in the upper airway, thus limiting its ability to identify potential risks.

There is limited high-level scientific evidence of the potential role of ultrasound in dynamic detection of upper airway changes during induction of anaesthesia. Furthermore, the data obtained do not indicate sufficiently whether these changes are associated with difficult airway. It is consequently critical that methodologically 
rigorous trials are performed to establish the use of ultrasound in assessment of difficult airway during anaesthetic induction and to provide new ideas and a theoretical basis for determining the correlation between dynamic airway changes and difficult airways.

We aim to address this issue in the study proposed here. Subjects will be recruited from the First Affiliated Hospital of Guangzhou University of Chinese Medicine, a tertiary teaching hospital in China. In this institute, nearly 20000 cases undergo operating room anaesthesia annually, $60 \%$ of which involve general anaesthesia; this can provide sufficient cases for addressing the critical clinical issues discussed above.

\section{METHODS AND ANALYSIS}

\section{Aim of the current study}

To evaluate changes in anterior cervical soft tissue thickness at five anatomical levels (hyoid bone, epiglottis, cricothyroid membrane, thyroid isthmus and suprasternal notch) in the upper airway, before and after induction of general anaesthesia.

To detect whether correlations exist between changes in ultrasound measurements and mask ventilation pressure, Cormack-Lehane score, demographic variables and clinical screening tests and to identify the usefulness of these measurements for assessment of a difficult airway.

\section{Design and setting}

The study is designed as a prospective observational single-blinded study, conducted in a single centre. The flowchart of this trial is provided in figure 1. The study will observe patients scheduled for elective surgery under general anaesthesia in the operating theatre of the First Affiliated Hospital of Guangzhou University of Chinese Medicine from February 2019 to January 2020.

\section{Sample size and study power}

Sample size is estimated based on a pilot study of 18 subjects. The change in the depth from the skin to the epiglottis before and after induction of anaesthesia in the pilot study was $0.22 \mathrm{~cm} \pm 0.55$ (mean $\pm \mathrm{SD})$. Assuming type I and type II errors of 0.05 and 0.10 , respectively, at least 66 subjects should be enrolled in this study. To adjust for attrition by dropout, 75 subjects will be recruited into the study.

\section{Participants}

Patients aged from 18 to 65 years, ASA physical status I-II and scheduled for elective surgery under general anaesthesia in operating room will be screened for eligibility. We will use no sex restriction. The inclusion and exclusion criteria are provided in table 1 . If any subject is thought to have allergic symptoms or haemodynamic changes, the corresponding treatment and tracheal intubation will be given immediately, and the participant will be withdrawn from the study. If face mask ventilation is inadequate, the Intubating laryngeal mask airway (ILMA) will be used to

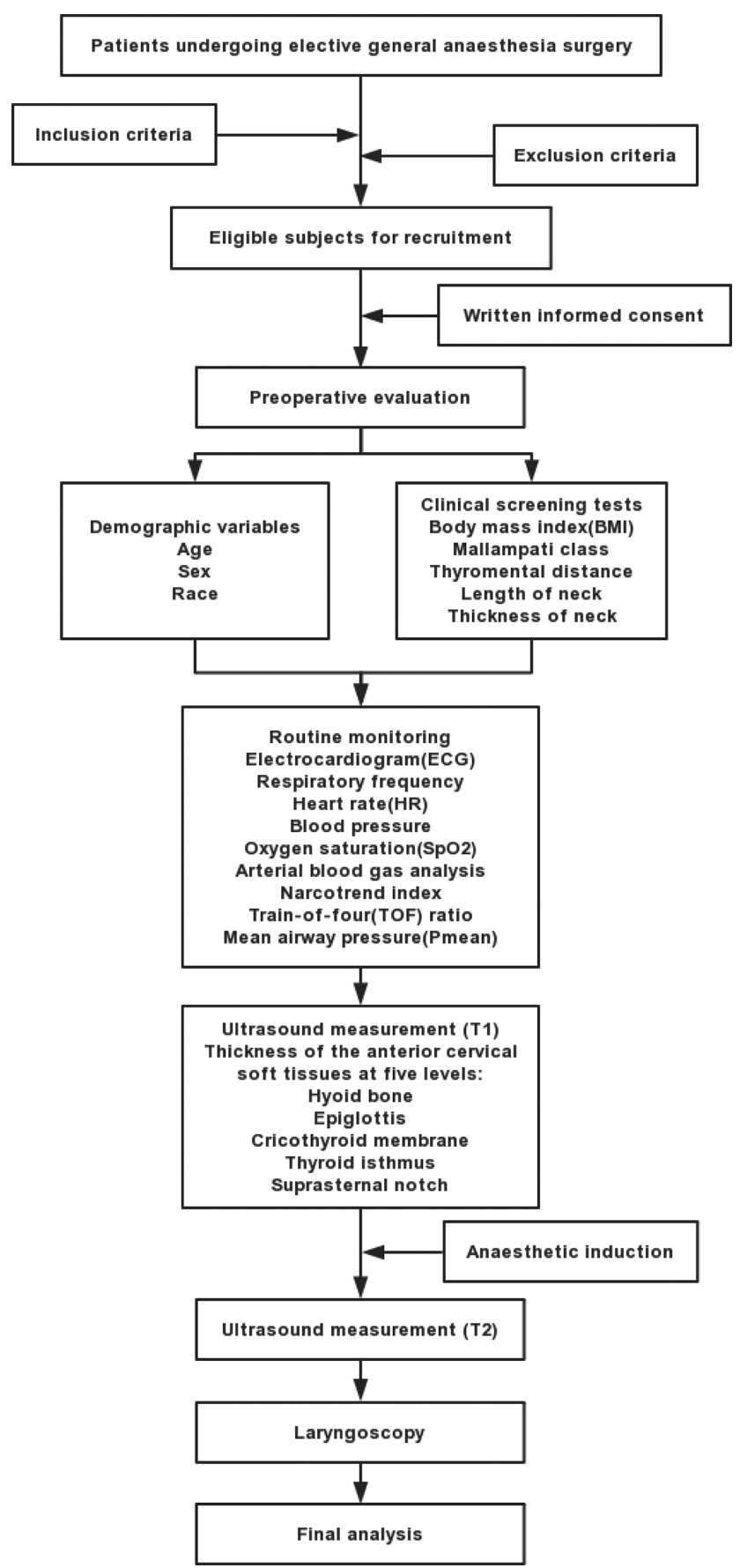

Figure 1 Flow chart of the study.

maintain ventilation. After sufficient oxygenation, fibreoptic-guided intubation through ILMA will be performed. If the Supraglottic airways (SGA) cannot maintain ventilation and oxygenation, cricothyroid membrane puncture will be performed immediately. In addition, patients with unanticipated difficult ventilation or intubation will be withdrawn from the study.

\section{Observations}

Ultrasound measurement

Before (T1) and after anaesthetic induction with loss of consciousness (T2), airway sonographic measurements (Navi series, Shenzhen Wisonic Medical Technologies, 
Table 1 Trial Inclusion and exclusion criteria

\begin{tabular}{|c|c|}
\hline Inclusion criteria & Exclusion criteria \\
\hline Age: 18-65years & $\begin{array}{l}\text { Pregnant women and } \\
\text { parturients }\end{array}$ \\
\hline ASA grade I or II & $\begin{array}{l}\text { Indications for awake and/ } \\
\text { or spontaneous breathing } \\
\text { airway management } \\
\text { technique }\end{array}$ \\
\hline $\begin{array}{l}\text { Undergoing elective surgery } \\
\text { under general anaesthesia }\end{array}$ & $\begin{array}{l}\text { Abnormal pharynx or } \\
\text { anatomy }\end{array}$ \\
\hline Appropriately NPO & $\begin{array}{l}\text { Preoperative Hct }<35 \% \text {; } \\
\mathrm{Hb}<110 \mathrm{~g} / \mathrm{L}\end{array}$ \\
\hline \multirow[t]{3}{*}{ Consent to participate } & $\begin{array}{l}\text { Severe cardiovascular or } \\
\text { cardiopulmonary diseases, } \\
\text { hepatic or renal dysfunction }\end{array}$ \\
\hline & Coagulation disorders \\
\hline & Refusal to participate \\
\hline
\end{tabular}

ASA, American Society of Anesthesiologists; $\mathrm{Hb}$, haemoglobin; Hct, haematocrit; NPO, Nil per os (nothing by mouth).

Shenzhen, China) will be performed by an attending anaesthesiologist, using a linear ultrasound transducer. Participants will be kept supine, in the sniffing position throughout the measurement procedure. ${ }^{31}{ }^{32}$ The probe will be placed in the submental region of the neck, in the midline, to measure the thickness of the anterior cervical soft tissues at five levels (hyoid bone (figure 2A), epiglottis (figure 2B), cricothyroid membrane (figure 2C), thyroid isthmus (figure 2D) and suprasternal notch (figure 2E)) in the axial plane. Measurement sites used during the first measurement will be marked to allow easier positioning of the probe at the same site in subsequent measurements. The images will be stored on the hard drive of the ultrasound machine.

\section{Airway pressure measurement}

After induction of anaesthesia, routine pressure-controlled face mask ventilation will be administered to the patient. Concomitantly, the mean airway pressure will be measured with a variable reluctance diaphragm pressure transducer (Datex-Ohmeda Aestiva 7100, GE Healthcare, Chicago, Illinois). Difficult mask ventilation will be recorded.

\section{Laryngoscopy}

When the muscle relaxes completely (train-of-four, TOF $1 / 40$ ), laryngoscopy will be performed by another attending anaesthetist who is blinded to the results of the ultrasound assessments. In accordance with a modified version of the Cormack-Lehane classification system, ${ }^{33} 34$ the Cormack-Lehane grades 1 or $2 \mathrm{a}$, grades $2 \mathrm{~b}$ or $3 \mathrm{a}$, and grades $3 \mathrm{~b}$ or 4 will be designated as easy, restricted and difficult laryngoscopies, respectively.
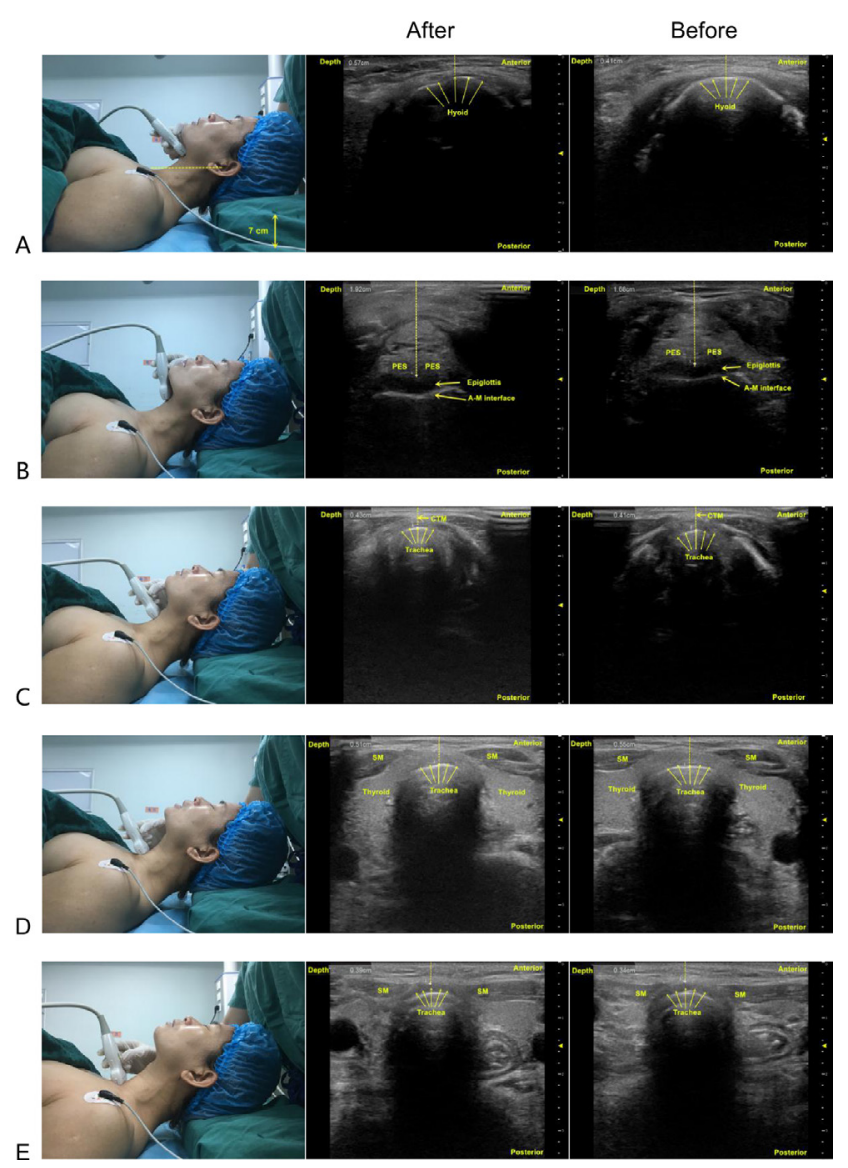

Figure 2 Ultrasonography axial views at the level of the hyoid bone $(A)$, epiglottis $(B)$, cricothyroid membrane $(C)$, thyroid isthmus (D) and suprasternal notch (E), before and after anaesthetic induction. Consent to publish the figure was obtained from the patient. A-M interface, air mucosa interface; CTM, cricothyroid membrane; PES, pre-epiglotic space; SM, strap muscles.

\section{Anaesthetic induction procedure}

Standard monitoring will be performed throughout anaesthesia to detect clinical vital signs and indicators related to the depth of anaesthesia (table 2). When the Narcotrend (MonitorTechnik, Bad Bramstedt, Germany) reaches stage $\mathrm{D} 2$, the second ultrasound measurement will be made. The induction will be performed as per standard practice of rapid sequence anaesthesia induction (see box 1$).^{35}$

Table 2 Standard monitoring

\begin{tabular}{ll}
\hline Clinical vital signs & Anaesthesia-related indicators \\
\hline ECG & Narcotrend stages and index \\
\hline Respiratory frequency & Train-of-four ratio \\
\hline Heart rate & Mean airway pressure \\
\hline $\begin{array}{l}\text { Blood pressure } \\
\text { Percutaneous oxygen } \\
\text { saturation }\end{array}$ & Arterial blood gas measurements \\
\hline
\end{tabular}




\section{Box 1 Anaesthetic induction procedure}

1. Preoxygenation treatment through a face mask for $3 \mathrm{~min}$.

2. Induction with intravenous propofol $1.5 \mathrm{mg} / \mathrm{kg}$.

3. Surgical analgesia with intravenous sufentanil; infusion at $0.5-1 \mu \mathrm{g} /$ $\mathrm{kg} / \mathrm{min}$, adjusted according to the Narcotrend (stage D2).

4. Neuromuscular blockade with intravenous rocuronium $0.6 \mathrm{mg} / \mathrm{kg}$.

5. Pressure-controlled facemask ventilation with a tidal volume of $8 \mathrm{~mL} / \mathrm{kg}$.

6. Endotracheal intubation with a direct laryngoscope.

\section{Outcome measurements}

The primary outcome is the changes in the thickness of the anterior cervical soft tissue between baseline and after anaesthetic induction. The ultrasound measurement will be used to evaluate thicknesses at five anatomical levels: hyoid bone, epiglottis, cricothyroid membrane, thyroid isthmus and suprasternal notch.

The secondary outcomes include the demographic variables, clinical screening tests, laryngoscopy results and anaesthesia-related indicators (table 3). Arterial blood gas measurements will be examined before and after anaesthetic induction.

\section{Recruitment and single blinding}

The subjects will be recruited from the surgical ward, and written informed consent will be obtained during the preoperative visit. The three attending anaesthesiologists will be blinded to each other's preoperative evaluation, ultrasound and laryngoscopy findings.

\section{Patient and public involvement}

The protocol of this study was developed and implemented through discussion with a patient and public involvement (PPI) group in face-to-face conversations and via messages, emails and phone calls. LS was a patient scheduled for orthopaedic surgery who served as a patient adviser. He was consulted on key aspects of the study design, including the recruitment and dissemination methods, consent form, image data acquisition and selection of the outcomes. The PPI representatives read the final protocol prior to the manuscript submission. In addition to the dissemination to clinicians and researchers through publication of professional clinical papers and delivery of academic speeches, key results of the trial will be disseminated via email to the participants to encourage further publicity with respect to the results.

\section{Statistical analysis}

Data will be analysed using the IBM Statistical Package for the Social Sciences (SPSS) V.20.0. The Shapiro-Wilks test will be used to first verify the normality of the sonographic data before and after anaesthetic induction. According to distribution sets, these changes will be analysed using a paired t-test or the Wilcoxon signed-rank test. Comparison of the area under the receiver-operating characteristic curves will determine the correlation between sonographic airway changes and the Cormack-Lehane grade, and will be used to assess optimal cut-off scores. The independent contribution of demographic variables, clinical screening tests and anaesthesia-related indicators (table 3) to airway changes will be assessed in a multivariate analysis using forward stepwise multiple linear regression. For all analyses, a $p$ value $<0.05$ will be considered statistically significant.

\section{ETHICS AND DISSEMINATION}

Written informed consent in both Chinese and English will be obtained from every participant prior to enrolment. The subjects can voluntarily withdraw from the trial at any time. Such withdrawal will not affect the medical intervention provided by the institution. Participants will be anonymised in the study by a medical identification code.

Table 3 Outcome measurements

Primary outcome

Sonographic measurements: thickness of anterior cervical soft tissue at five levels

Hyoid bone Epiglottis Cricothyroid membrane Thyroid isthmus Suprasternal notch

Secondary outcomes

\begin{tabular}{|c|c|c|c|}
\hline Demographic variables & $\begin{array}{l}\text { Clinical screening } \\
\text { Tests }\end{array}$ & Laryngoscopy results & $\begin{array}{l}\text { Anaesthesia-related } \\
\text { indicators }\end{array}$ \\
\hline Age & Body mass index (BMI) & Cormack-Lehane grade & Mean airway pressure \\
\hline Sex & Mallampati class & & $\mathrm{SpO}_{2}$ \\
\hline Race & Thyromental distance & & $\begin{array}{l}\text { Arterial blood oxygen } \\
\text { and carbon dioxide } \\
\mathrm{PaO} 2, \mathrm{PaCO} 2\end{array}$ \\
\hline
\end{tabular}

Length of neck

Thickness of neck

$\mathrm{PaO}_{2}$, partial pressure of oxygen; $\mathrm{PaCO} 2$, partial pressure of carbon dioxide; $\mathrm{SpO}_{2}$, peripheral pulse oximetry. 
The First Affiliated Hospital of Guangzhou University of Chinese Medicine is one of the four national airway management training bases of the Chinese Association of Anaesthesiologists. As an academic and training centre, the department of anaesthesiology provides comprehensive operative services to a large population. It also serves as the primary teaching site to provide systematic, standardised and normalised training for medical staff. Many international conferences and training courses have been held in our hospital in conjunction with well-known universities worldwide. Since 2008, more than 1600 anaesthesiologists, emergency doctors, intensive care unit doctors and respiratory physicians from 29 provinces and municipalities in China have participated in an airway management training programme. In 2018, the director of the department delivered a speech regarding the status quo of airway management in China at the conference of the American Association of Airway Management. These all provided sufficient preconditions and conditions for validation and promotion of new technologies.

To date, there has been no previous ultrasonographic study that has observed the morphological changes in the upper airway before and after induction of general anaesthesia. Our study will involve monitoring these changes and analysing the correlation between changes in the upper airway and difficult airway by regression analysis, to provide a new basis for evaluation of difficult airways and to explore new and more effective methods to address this problem. It will provide novel indicators that can form a basis for new clinical guidelines in airway assessment. The findings will be published in professional journals and in peer-reviewed journals. They will be presented at seminars and scientific conferences. The key audience for dissemination will be anaesthesiologists, emergency physicians, intensive care unit doctors and sleep medicine physicians in China as well as internationally.

Acknowledgements We wish to acknowledge Lei Su and the patient and public involvement group who made public suggestions for the study design and dissemination.

Contributors WM conceptualised and designed the study, wrote funding applications and provides professional consultation, assists with methodology and administers the project. FD drafted, reviewed and edited this manuscript, and will help to collect and analyse the data. YW performed the pilot study and collected data for estimating the sample size and reviewed and edited this manuscript. XW and $\mathrm{HZ}$ performed the pilot study and collected data for estimating the sample size and will assist in further data collection.

Funding The trial was supported by the Guangzhou Science and Technology Bureau (Grant number: 201707010297) and National Natural Science Foundation of China (Grant numbers: 81503663 and 81673922).

Competing interests None declared.

Patient consent for publication Not required.

Provenance and peer review Not commissioned; externally peer reviewed.

Open access This is an open access article distributed in accordance with the Creative Commons Attribution Non Commercial (CC BY-NC 4.0) license, which permits others to distribute, remix, adapt, build upon this work non-commercially, and license their derivative works on different terms, provided the original work is properly cited, appropriate credit is given, any changes made indicated, and the use is non-commercial. See: http://creativecommons.org/licenses/by-nc/4.0/.

\section{REFERENCES}

1. Sorbello M, Afshari A, De Hert S. Device or target? A paradigm shift in airway management: Implications for guidelines, clinical practice and teaching. Eur J Anaesthesiol 2018;35:811-4.

2. Cormack RS, Lehane J. Difficult tracheal intubation in obstetrics. Anaesthesia 1984;39:1105-11.

3. Cook TM, Woodall N, Frerk C. Fourth National Audit Project. Major complications of airway management in the UK: results of the Fourth National Audit Project of the Royal College of Anaesthetists and the Difficult Airway Society. Part 1: anaesthesia. $\mathrm{Br} J$ Anaesth 2011;106:617-31.

4. Cook TM, MacDougall-Davis SR. Complications and failure of airway management. Br J Anaesth 2012;109 Suppl 1:i68-i85.

5. Hillman DR, Platt PR, Eastwood PR. The upper airway during anaesthesia. Br J Anaesth 2003;91:31-9.

6. Deegan PC, Mulloy E, McNicholas WT. Topical oropharyngeal anesthesia in patients with obstructive sleep apnea. Am J Respir Crit Care Med 1995;151:1108-12.

7. Alkire MT, Haier RJ, Fallon JH. Toward a unified theory of narcosis: brain imaging evidence for a thalamocortical switch as the neurophysiologic basis of anesthetic-induced unconsciousness. Conscious Cogn 2000;9:370-86.

8. Hillman DR, Walsh JH, Maddison KJ, et al. Evolution of changes in upper airway collapsibility during slow induction of anesthesia with propofol. Anesthesiology 2009;111:63-71.

9. Suto $\mathrm{Y}$, Matsuo T, Kato T, et al. Evaluation of the pharyngeal airway in patients with sleep apnea: value of ultrafast MR imaging. AJR Am J Roentgenol 1993;160:311-4.

10. Ciscar MA, Juan G, Martínez V, et al. Magnetic resonance imaging of the pharynx in OSA patients and healthy subjects. Eur Respir $J$ 2001;17:79-86.

11. Frova G, Sorbello M. Algorithms for difficult airway management: a review. Minerva Anestesiol 2009;75:201-9.

12. Apfelbaum JL, Hagberg CA, Caplan RA, et al. Practice guidelines for management of the difficult airway: an updated report by the American Society of Anesthesiologists Task Force on Management of the Difficult Airway. Anesthesiology 2013;118:251-70.

13. Roth D, Pace NL, Lee A, et al. Airway physical examination tests for detection of difficult airway management in apparently normal adult patients. Cochrane Database Syst Rev 2018;5:CD008874.

14. Nørskov AK, Rosenstock CV, Wetterslev J, et al. Diagnostic accuracy of anaesthesiologists' prediction of difficult airway management in daily clinical practice: a cohort study of 188064 patients registered in the Danish Anaesthesia Database. Anaesthesia 2015;70:272-81.

15. Lee A, Fan LT, Gin T, et al. A systematic review (meta-analysis) of the accuracy of the Mallampati tests to predict the difficult airway. Anesth Analg 2006;102:1867-78.

16. Vannucci A, Cavallone LF. Bedside predictors of difficult intubation: a systematic review. Minerva Anestesiol 2016;82:69-83.

17. Moorthy SS, Gupta S, Laurent B, et al. Management of airway in patients with laryngeal tumors. J Clin Anesth 2005;17:604-9.

18. Rosenblatt W, lanus Al, Sukhupragarn W, et al. Preoperative endoscopic airway examination (PEAE) provides superior airway information and may reduce the use of unnecessary awake intubation. Anesth Analg 2011;112:602-7.

19. Döhlemann C, Mantel K, Vogl TJ, et al. Pulmonary sling: morphological findings. Pre- and postoperative course. Eur J Pediatr 1995;154:2-14.

20. Wani TM, Bissonnette B, Rafiq Malik M, et al. Age-based analysis of pediatric upper airway dimensions using computed tomography imaging. Pediatr Pulmonol 2016;51:267-71.

21. Baroni RH, Feller-Kopman D, Nishino M, et al. Tracheobronchomalacia: comparison between end-expiratory and dynamic expiratory CT for evaluation of central airway collapse. Radiology 2005;235:635-41.

22. Feng $\mathrm{Y}$, Keenan BT, Wang S, et al. Dynamic upper airway imaging during wakefulness in obese subjects with and without sleep apnea. Am J Respir Crit Care Med 2018;198:1435-43.

23. Isaiah A, Kiss E, Olomu $P$, et al. Characterization of upper airway obstruction using cine MRI in children with residual obstructive sleep apnea after adenotonsillectomy. Sleep Med 2018;50:79-86.

24. Faust RA, Remley KB, Rimell FL. Real-time, cine magnetic resonance imaging for evaluation of the pediatric airway. Laryngoscope 2001;111:2187-90.

25. El-Boghdadly K, Onwochei DN, Millhoff B, et al. The effect of virtual endoscopy on diagnostic accuracy and airway management 
strategies in patients with head and neck pathology: a prospective cohort study. Can J Anaesth 2017;64:1101-10.

26. Guay J, Kopp S. Ultrasonography of the airway to identify patients at risk for difficult tracheal intubation: Are we there yet? J Clin Anesth 2018:46:112-5.

27. Falcetta S, Cavallo S, Gabbanelli V, et al. Evaluation of two neck ultrasound measurements as predictors of difficult direct laryngoscopy: A prospective observational study. Eur J Anaesthesiol 2018:35:605-12.

28. Detsky ME, Jivraj N, Adhikari NK, et al. Will this patient be difficult to intubate?: the rational clinical examination systematic review. JAMA 2019;321:493-503.

29. Abbey NC, Block AJ, Green D, et al. Measurement of pharyngeal volume by digitized magnetic resonance imaging. Am Rev Respir Dis 1989;140:717-23.

30. White DP. Pathogenesis of obstructive and central sleep apnea. Am J Respir Crit Care Med 2005;172:1363-70.
31. Mendonca C, Ungureanu N, Nowicka A, et al. A randomised clinical trial comparing the 'sniffing' and neutral position using channelled $\left(\right.$ KingVision $\left.^{\circledR}\right)$ and non-channelled $\left(\mathrm{C}-\mathrm{MAC}^{\circledR}\right)$ videolaryngoscopes. Anaesthesia 2018;73:847-55.

32. Greenland KB, Edwards MJ, Hutton NJ. External auditory meatussternal notch relationship in adults in the sniffing position: a magnetic resonance imaging study. Br J Anaesth 2010;104:268-9.

33. Yentis SM. Laryngoscopy grades. Anaesthesia 1999;54:1221-2.

34. Cook TM. A new practical classification of laryngeal view. Anaesthesia 2000;55:274-9.

35. Sajayan A, Wicker J, Ungureanu N, et al. Current practice of rapid sequence induction of anaesthesia in the UK - a national survey. $\mathrm{Br} \mathrm{J}$ Anaesth 2016;117(Suppl 1):i69-i74. 